\title{
Response of lignophenols for oxidation and reduction systems
}

\author{
Shimpei HORII and Masamitsu FUNAOKA \\ Graduate School of Bioresources, Mie University, SORST JST, Tsu, Japan \\ Fax: 059-231-9521, e-mail: funaoka@bio.mie-u.ac.jp
}

Lignin is one of the most abundant renewable materials. It has perfect biodegradability in soil. This biodegradation proceeds oxidatively and reductively. In order to utilize along the material flow in the ecosystem, it is important to obtain information for oxidative and reductive responses of lignophenols, which were synthesized through the phase-separation system. Lignocresol was synthesized from Hinoki cypress (Chamaecyparis obtusa) through the phase-separation system with $72 \% \mathrm{H}_{2} \mathrm{SO}_{4}$ and $p$-cresol. Responses under both oxidation and reduction environments were estimated by FT-IR, TGA and TMA. Both lignocresol and wood meals were oxidized with sodium periodate. FT-IR spectra of oxidized lignocresol indicated the formation of muconic acid type structures. FT-IR spectra of lignocresol derived from oxidized wood meals indicated the phenolation of conjugated carbonyl structures. Thermal stability of lignophenols was improved by the reduction treatments with sodium borohydride.

Key words: Lignin, Phase-separation system, Lignophenol, Oxidation, Reduction

\section{INTRODUCTION}

Lignin is one of the most abundant renewable materials on the earth, accounting for approximately $25-35 \%$ of the organic matrix of lignocellulosic biomass. It has high potential as a feedstock for producing chemicals, fuels and materials. Lignin has been mainly used as an energy source in pulp and paper industries. During pulping process at high temperature and pressure, structures of lignin become complex. These random structures make the utilization difficult as a functional polymer.

However, selective structural control of lignin can be achieved through the phase-separation system, which has been developed by Funaoka since 1988 [1-3]. Lignocellulosics are rapidly separated to water-soluble saccharides and lignin derivatives with concentrated acid and phenol derivatives under ordinary pressure at room temperature through the system. The separated lignin derivatives (lignophenol) have 1,1-bis(aryl)propane type structures. Lignophenols also have several structural characteristics. They are highly phenolic and very lightly colored etc. Additionally, lignophenols were designed to be able to control the functionalities such as molecular weight. Lignophenols have been expected to be effectively utilized as alternative resources to fossil materials.

In order to utilize lignin along a material flow in the ecosystem, it is important to obtain information for oxidative and reductive responses. Lignin is biodegraded in soil with creating various functions such as adsorption of metallic ions or proteins. That degradation proceeds oxidatively and reductively.

In this work, periodate oxidation and borohydride reduction were applied to wood meals and lignophenols, and the structural features of the resulting lignins were discussed in the point of view of functionality control for successive utilization. Generally periodate oxidation has been used for estimation of phenolic hydroxyl groups in lignin $[4,5]$. One mole of methanol per mole of phenolic hydroxyl group is released during the oxidation. At this time, aromatic ring of lignin with phenolic hydroxyl group was converted to muconic acid type structures via $o$-quinone structure. Borohydride reduction has been investigated for bleaching of pulp $[6,9]$. The combination of oxidation and reduction might enable selective functionality control of lignophenol.

\section{EXPERIMENTAL}

\subsection{Synthesis of lignophenol}

The chips of Hinoki cypress (Chamaecyparis obtusa) were milled to 60 mesh. Extractives in the wood meals were removed with benzene / ethanol $=2 / 1(\mathrm{v} / \mathrm{v})$ using Soxhlet system for $72 \mathrm{hrs}$. Lignocresol (LC) was synthesized through the phase-separation system, using two-step method (process II) (Fig. 1). The wood meals $(50 \mathrm{~g})$ were immersed in acetone solution of $p$-cresol with concentration of $3 \mathrm{~mol} /$ phenylpropane units $\left(\mathrm{C}_{9}\right.$

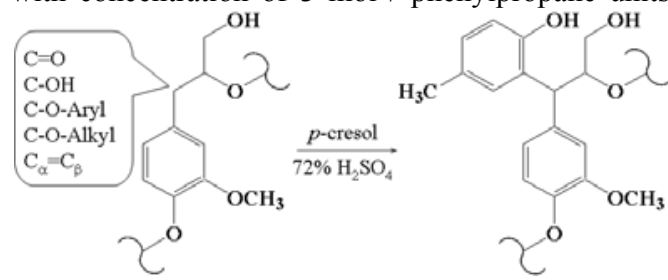

Fig. 1 Synthesis of lignophenols through the phaseseparation system with $p$-cresol and $72 \% \mathrm{H}_{2} \mathrm{SO}_{4}$.

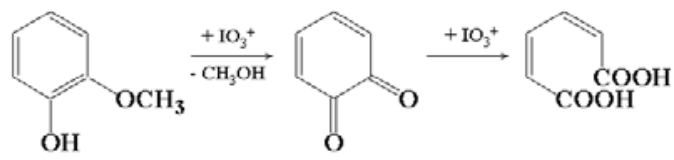

Fig. 2 Periodate oxidation of lignin model.

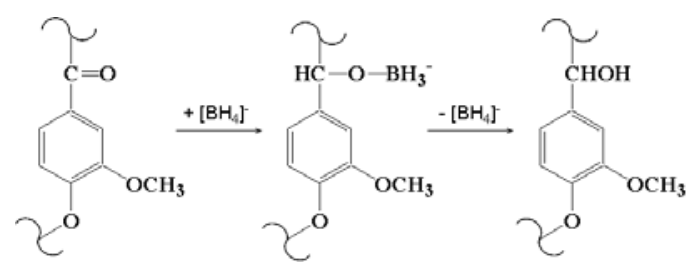

Fig. 3 Borohydride reduction of lignin. 
units). After evaporating acetone, $72 \% \mathrm{H}_{2} \mathrm{SO}_{4}$ was poured into the wood meals at $30^{\circ} \mathrm{C}$. Then, the mixture was stirred vigorously for $60 \mathrm{~min}$. Then, the mixture was poured into $2 \mathrm{~L}$ of de-ionized water with vigorously stirring. Then the precipitation was washed until neutral. After drying, the precipitates were dissolved in acetone, and insoluble materials were removed by centrifugation and filtration. The acetone solution was concentrated under reduced pressure and added dropwise to excess amount of diethyl ether with stirring. The precipitated lignocresol was collected by centrifugation. After evaporating and drying on $\mathrm{P}_{2} \mathrm{O}_{5}$, lignocresol was obtained.

\subsection{Periodate oxidation}

Periodate oxidation was carried out, according to the procedure by Funaoka (Fig. 2) [2]. Oxidation of wood meals: A saturated sodium periodate $\left(\mathrm{NaIO}_{4}\right)$ solution kept at $4^{\circ} \mathrm{C}(15 \mathrm{~mL} / \mathrm{g})$ was added to wood meals. The suspension was homogenized and placed in a refrigerator at $4^{\circ} \mathrm{C}$ with occasional stirring for $48 \mathrm{hrs}$. After the treatment, the materials were filtered and washed with cold de-ionized water. After freeze-drying and drying on $\mathrm{P}_{2} \mathrm{O}_{5}$, oxidized wood meals were obtained.

Oxidation of lignocresol: Lignocresol was dissolved in glacial acetic acid $(1 \mathrm{~mL} / 100 \mathrm{mg})$, to which the sodium periodate $\left(\mathrm{NaIO}_{4}\right)$ solution $(15 \mathrm{~mL} / 100 \mathrm{mg})$ was added. The reaction mixture was kept with occasional stirring at $4^{\circ} \mathrm{C}$ for $72 \mathrm{hrs}$. Then, it was added dropwise to an excess amount of cold water with vigorous stirring and the precipitated oxidized lignocresols were collected by centrifugation and washed with cold water. After freeze-drying followed by drying on $\mathrm{P}_{2} \mathrm{O}_{5}$, oxidized lignocresol (OLC) was obtained.

\subsection{Synthesis of lignophenols from oxidized wood meals}

Lignocresol was synthesized from oxidized wood meals through the phase-separation system, using one-step method. $p$-Cresol $(10 \mathrm{~mL} / \mathrm{g}$ wood $)$ was added to wood meals with stirring. After $10 \mathrm{~min}, 72 \% \mathrm{H}_{2} \mathrm{SO}_{4}(20 \mathrm{~mL} / \mathrm{g}$ wood) was added to the mixture and the vigorous stirring was continued at room temperature for $60 \mathrm{~min}$. The separated cresol phase was added dropwise to an excess of diethyl ether with vigorous stirring. The precipitates were dissolved in acetone, and insoluble materials were removed by centrifugation and filtration. The acetone solution was concentrated under reduced pressure and added dropwise to an excess of diethyl

Table I Yields of lignophenols.

\begin{tabular}{lcc}
\hline & \multicolumn{2}{c}{ Yields } \\
\cline { 2 - 3 } & (\%) of wood meal & (\%) of Klason lignin \\
\hline LC & 27.3 & 93.3 \\
OLC & 25.4 & 86.6 \\
LCO & 37.7 & 128.7 \\
RLC & 24.1 & 82.1 \\
ROLC & 22.7 & 77.3 \\
RLCO & 26.9 & 91.9 \\
\hline
\end{tabular}

* Klason lignin means the amount of lignin in the plant. ether with stirring. The precipitated lignocresol was collected by centrifugation. After evaporating and drying on $\mathrm{P}_{2} \mathrm{O}_{5}$, lignocresol derived from oxidized wood meals (LCO) was obtained.

\subsection{Sodium borohydride reduction}

Lignocresol, oxidized lignocresol and lignocresol derived from oxidized wood meals were dissolved in 0.1 $\mathrm{N} \mathrm{NaOH}$ under a nitrogen atmosphere, to which sodium borohydride $\left(\mathrm{NaBH}_{4}\right)$ was added (Fig. 3). The reaction mixture was kept with occasional stirring at room temperature for $72 \mathrm{hrs}$. Then, it was acidified to $\mathrm{pH} 2$ with $1 \mathrm{~N} \mathrm{HCl}$. The precipitates were collected by centrifugation, washed until neutral. After freeze-drying following by drying on $\mathrm{P}_{2} \mathrm{O}_{5}$, reduced lignocresol (RLC), reduced oxidized lignocresol (ROLC) and reduced lignocresol derived from oxidized wood meals (RLCO) was obtained.

\subsection{Alkaline treatment of lignophenol}

Lignocresol was dissolved in $0.1 \mathrm{~N} \mathrm{NaOH}$ under a nitrogen atmosphere. The reaction mixture was kept with occasional stirring at room temperature for $72 \mathrm{hrs}$. Then, it was acidified to $\mathrm{pH} 2$ with $1 \mathrm{~N} \mathrm{HCl}$. The precipitates (ALC) were collected by centrifugation and washed until neutral.

\subsection{Analytical methods}

The structures of lignophenols were characterized by Fourier Transformation Infra-Red spectroscopy (FT-IR), Thermogravimetry analysis (TGA) and Thermo Mechanical Analysis (TMA). FT-IR spectroscopy was carried out on a Spectrum GX (Perkin Elmer Co.), using the $\mathrm{KBr}$ pellet technique for sample preparation. TGA was carried out by TG/DTA-6200 (SII Inc.) at a rate $2^{\circ} \mathrm{C}$

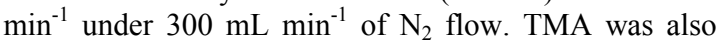
carried out by TMA-SS (SII Inc.) in the temperature range $50-280^{\circ} \mathrm{C}$ at a rate of $2^{\circ} \mathrm{C} \mathrm{min}^{-1}$, using penetrating technique for a measurement.

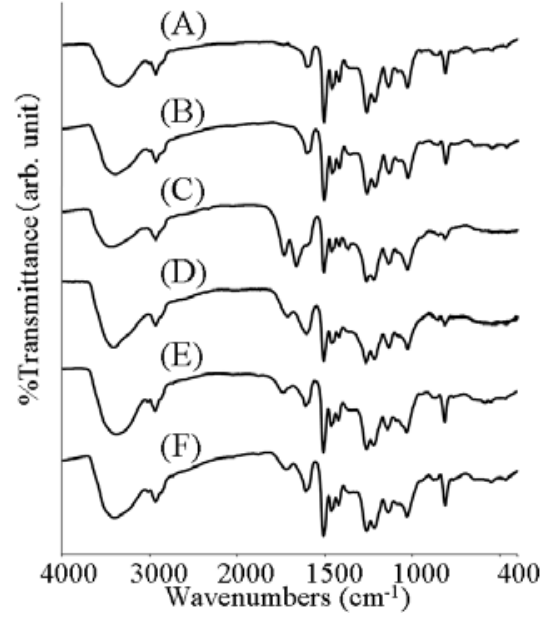

Fig. 4 FT-IR spectra of lignophenols. (A) Lignocresol; LC, (B) Reduced lignocresol; RLC, (C) Oxidized lignocresol; OLC, (D) Reduced oxidized lignocresol; ROLC, (E) Lignocresol derived from oxidized wood meals; LCO, (F) Reduced lignocresol derived from oxidized wood meals; RLCO 


\section{RESULTS AND DISCUSSION}

\subsection{Yields of lignophenols}

Yields of lignophenols were shown in Table 1. LC was effectively synthesized from Hinoki cypress wood meals. OLC was obtained in $93.3 \%$ yield from LC. In contrast, LCO was obtained in $128.7 \%$ yield based on Klason lignin. This would be attributed to large amount of introduced $p$-cresol. Through the phase-separation treatment after the oxidation, the phenolation for conjugated structures in muconic acid type structures occurred. However, LCO includes adsorbed $p$-cresol. RLC, ROLC, RLCO were obtained in about $88 \%, 89 \%$ and $71 \%$ yields, respectively. $p$-Cresol adsorbed on LCO would be washed away during the reduction treatment in the alkaline solution.

\subsection{Structural characterization of lignophenols}

FT-IR spectrum of LC had a sharp peak at $815 \mathrm{~cm}^{-1}$, assigned to $\mathrm{C}-\mathrm{H}$ deformations (two adjacent) of cresol (Fig. 4A) [2]. The spectrum of RLC reveals the presence of less carbonyl structures in LC (Fig. 4B). OLC had smaller peaks at $815 \mathrm{~cm}^{-1}$ and $1600 \mathrm{~cm}^{-1}$ (Fig. 4C). Moreover, it had a peak assigned to conjugated carbonyl group at $1660 \mathrm{~cm}^{-1}$. These suggested that muconic acid type structures were formed by the cleavage of aromatic rings. The peak at $1740 \mathrm{~cm}^{-1}$ indicated that OLC had unconjugated carbonyl groups in $\mathrm{C} \gamma$ position. On the other hand, LCO had weak absorption around $1660 \mathrm{~cm}^{-1}$ and sharp absorption at $815 \mathrm{~cm}^{-1}$, indicating the introducing of $p$-cresol to conjugated carbonyl structures By the reduction of OLC and $\mathrm{LCO}$ with $\mathrm{NaBH}_{4}$, only a weak band remains around $1720 \mathrm{~cm}^{-1}$ due to the slow reactivity of the unconjugated carbonyl groups (Fig. 4D, F) $[7,8,9]$.

\subsection{Thermal properties of lignophenols}

The results of TGA were shown in Fig. $5 . \mathrm{Td}_{5}$ and $\mathrm{Td}_{10}$ of LC were observed at 175 and $242^{\circ} \mathrm{C}$, respectively (Fig. 5A). On the other hand, OLC showed $191^{\circ} \mathrm{C}$ and $233^{\circ} \mathrm{C}$ respectively. Due to molecular associate of carboxyl groups in muconic acid type structures, thermal stability was improved (Fig. 5B). However, the improvement could be due to the loss of the certain parts during oxidation treatment, which is the lower resistant fraction under the heat condition. The weight loss of LCO was observed at lower temperatures than LC (Fig. 5C). This is attributed to adsorption of $p$-cresol at the surface of LCO structures. Therefore LCO seems to have higher adsorption ability. After the reduction with $\mathrm{NaBH}_{4}$ in alkaline solution, thermal stabilities of LC, OLC and LCO were improved drastically (Fig. 5D, E, F).

The results of TMA were shown in Fig. 6. TMA profiles showed that solid-liquid transition temperature of LC was $157.7^{\circ} \mathrm{C}$ (Fig. 6A). This flux indicated that $\mathrm{LC}$ is a liner-type polymer. OLC showed no fluidity, indicating that thermal stability was improved as well as the result of TGA (Fig. 6B). LCO has the transition point at $161^{\circ} \mathrm{C}$ and remarkable swelling during the flux (Fig. 6C). This swelling was due to the increase of viscosity. The increase of viscosity is due to the improvement of the interaction among molecules. Higher adsorption ability of LCO can be accounted by this improvement. In agreement with the results of TGA, reduced lignophenols have higher thermal stability than before.
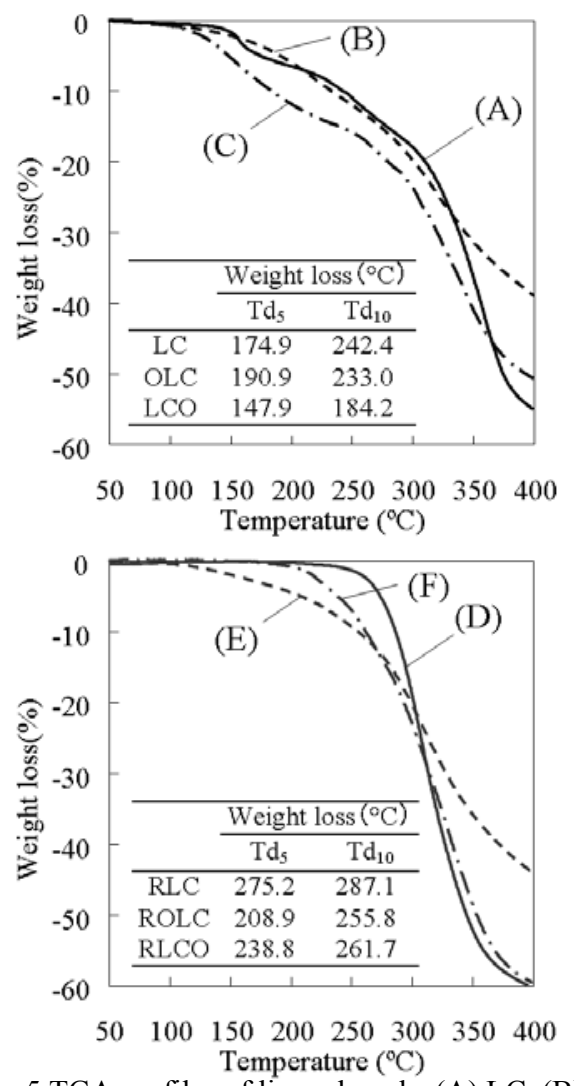

Fig. 5 TGA profiles of lignophenols. (A) LC, (B) OLC, (C) LCO, (D) RLC, (E) ROLC, (F) RLCO
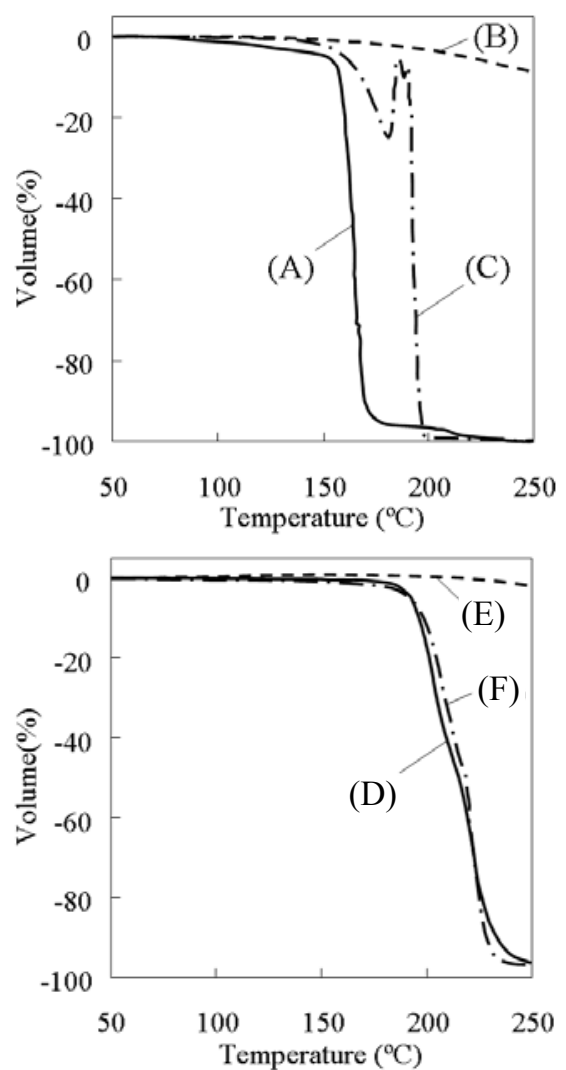

Fig. 6 TMA profiles of lignophenols. (A) LC, (B) OLC, (C) LCO, (D) RLC, (E) ROLC, (F) RLCO 


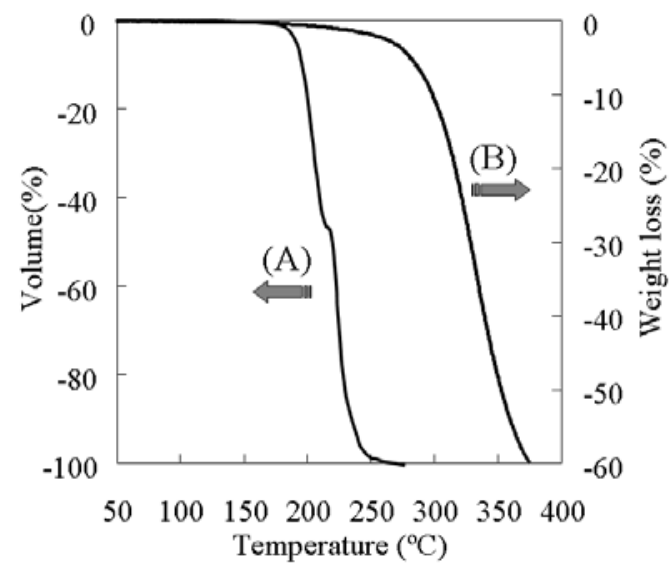

Fig. 7 TGA and TMA profiles of ALC. (A) TMA (B) TGA
(A)<smiles>CCOC(C=O)C(c1cccc(=O)o1)c1cc(C)cc(=O)o1</smiles>

(C)<smiles>CCC=C(CO)C(C1=CC(C)=CCC1)C(CO)OC</smiles>

(B)<smiles>CCOC(C=O)C(C1=CC(=O)CC(Oc2ccc(C)cc2)C1=O)c1cc(C)ccc1O</smiles>

(D)

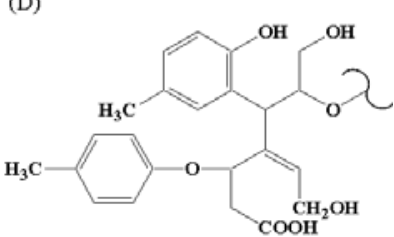

Fig. 8 Proposed structures of lignophenols after oxidation and reduction treatments. (A) OLC, (B) LCO, (C) ROLC, (D) RLCO

This improvement of thermal stability would be affected by the amount of hydroxyl groups. Probably, reduced lignophenols have high hydroxyl contents. Its hydroxyl groups have some hydrogen bonds, which improve the interactions among molecules. However, alkaline treatments also effect on thermal stability. TGA and TMA profiles of ALC were shown in Fig. 7. $\mathrm{Td}_{5}$ and $\mathrm{Td}_{10}$ were observed at 281.1 and $298.5^{\circ} \mathrm{C}$ respectively. The solid-liquid transition temperature was $194.2^{\circ} \mathrm{C}$. The improvements of thermal stability were in the same range with reduced lignophenols. By alkaline treatment, the linkage at $\mathrm{C} \alpha$ position such as benzyl aryl ether bond can be cleaved. The low-molecular lignin fractions eliminated during alkaline treatment would behave as an internal plasticizer in lignophenol without alkaline treatment. Therefore, the cleavages of the linkage at $\mathrm{C} \alpha$ position would improve thermal stability.

\subsection{Proposed structures of lignophenols}

Periodate oxidation of LC cleaves the aromatic ring, and then muconic acid type structures are formed (Fig. 8A). Through the phase-separation treatment after the oxidation, conjugated structures are disappeared by the introducing $p$-cresol (Fig. 8B). The main structures of RLC are similar to LC because of a small amount of carbonyl structures. After the borohydride reduction, carbonyl groups in OLC and LCO are converted to alcohol (Figs. 8C and D, respectively). Especially, conjugated carbonyl groups are reduced rapidly than unconjugated ones.

\subsection{Potential of these materials as the feedstock}

Lignophenols have been investigated as the feedstock for bio-based products such as lignophenol-fiber complex [3], dye-sensities solar cell [10], bioreactor with enzyme-immobilized ability [3] etc. A unique characteristic of OLC is to have the strong hydrogen bonds due to many carboxyl groups, which can be applied as the reinforcement such as adhesive reagent. LCO has a high adsorption ability. This is expected the application as controlled-release formulation. RLC, ROLC and RLCO have high thermal stability. These would be utilized as molded materials with thermal process.

\section{CONCLUSION}

The structural characteristics of lignophenols treated under the oxidative and reductive environments were mainly discussed with FT-IR. After oxidation, OLC had muconic acid type structures. In the results by TGA and TMA, these high thermal stabilities were observed. This is due to hydrogen bonds between carboxylic groups. LCO was obtained in higher yield from oxidized wood meals. This is attributed to a high content of $p$-cresol. The interactions between the molecules of LCO were improved. Reduction treatments in alkaline solution provided higher thermal stability. This would be due to the increase of hydroxyl group. Alkaline treatments also improved the thermal stability.

Oxidation and reduction treatments of lignophenols create new functions. These results indicated that these methods are effective for selective functionality control along the material flow in the ecosystem. These methods have the potential as new application of lignophenols.

\section{ACKNOWLEDGEMENT}

We thank Dr. Nonaka for helpful discussion.

\section{REFERENCES}

[1] M. Funaoka and I. Abe, Tappi journal, 72, 145-149 (1989)

[2] M. Funaoka and S. Fukatsu, Holzforschung, 50, 245-252 (1996)

[3] M. Funaoka, Polymer International, 47, 277-290 (1998)

[4] E. Adler and S. Hernestam, Acta Chem. Scand., 9, 319-334 (1955)

[5] E. Adler, S. Hernestam and I. Wallden, Svensk Papperstidn., 61, 641-647 (1958)

[6] W. C. Mayer and C. P. Donofrio, Pulp and Paper Magazine of Canada, 59, 157-163, 166 (1958)

[7] E. Adler and J. Marton, Acta Chem. Scand., 13, 75-96 (1959)

[8] J. Marton, E. Adler and K. I. Persson, Acta Chem. Scand., 15, 384-392 (1961)

[9] S. Y. Lin and K. P. Kringstad, Tappi, 53, 1675-1677 (1970)

[10] M. Aoyagi and M. Funaoka, J. Photochem. Photobiol. A Chem., 164, 53-60 (2004)

(Received December 12, 2008;Accepted September 16, 2009) 